\title{
Acompanhamento do Desempenho das Atribuições de Tutores a Distância: Método e Ferramenta
}

\author{
Carlos Henrique Leitão Cavalcante ${ }^{* 1}$, Verônica Lima Pimentel de Sousa ${ }^{2}$, Paulo \\ Benicio Melo de Sousa ${ }^{3}$
}

\begin{abstract}
1. Mestrando. Mestrado Profissional em Computação Aplicada (MPCOMP), Universidade Estadual do Ceará, Instituto Federal de Educação, Ciência e Tecnologia do Ceará (IFCE), Av. 13 de Maio, 2081 - Benfica, Fortaleza-CE-Brasil. henriqueleitao@gmail.com

2. Professora/Pesquisadora, Instituto Federal de Educação, Ciência e Tecnologia do Ceará (IFCE). Av. 13 de Maio, 2081 - Benfica, Fortaleza-CE-Brasil. veronica.pimentel@gmail.com

3. Professor/Pesquisador, Faculdade Metropolitana da Grande Fortaleza (FAMETRO). Rua Conselheiro Estelita, 500 - Centro, Fortaleza-CE-Brasil. paulo.benicio@gmail.com
\end{abstract}

\section{Resumo}

Entre os diversos papéis dos atores da Educação a Distância, destaca-se o papel desempenhado pelo tutor a distância, responsável por realizar o acompanhamento acadêmico, por reforçar o conteúdo e tirar dúvidas dos alunos na disciplina. Dessa forma, é de grande importância para a qualidade do ensino a distância o monitoramento, o controle e o acompanhamento de suas atribuições. Esse trabalho de supervisão da tutoria é necessário, pois visa corrigir problemas inerentes ao papel dos tutores, evitando prejuízos ao processo de ensino e aprendizagem nos cursos a distância. O estudo apresentado foi aplicado em 81 tutorias de um curso do programa Universidade Aberta do Brasil (UAB) em uma Instituição de Ensino Superior (IES). Nesse sentido, esta pesquisa possibilitou a classificação das atribuições de um tutor a distância, a delimitação de quais atribuições seriam monitoradas e um processo de definição de premissas e métricas de desempenho para cada uma dessas atribuições. Esse processo serviu de base para a criação de um mecanismo computacional, denominado MDTMoodle, que proporcionou a visualização do desempenho dos tutores a distância através de semáforos.

Palavras-chave: Educação a distância; Tutoria a distância; Acompanhamento de tutoria. 


\title{
Monitoring the Efficiency on Distance Tutors Roles: a method and a tool
}

\begin{abstract}
Among the various roles of the actors in Distance Learning, it is worth highlighting the role played by the tutor, who is responsible for performing academic monitoring, enhancing content, and taking questions from students on the subject matter. Therefore, monitoring, control, and accompaniment of tutors are very important for the quality of distance education. These will correct the problems, avoiding losses in the teaching and learning process. This work has been applied on 81 tutored online courses of a Higher Education Institution. In this context, the research has enabled a classification of the tutor's assignments, the boundaries of which ones would be monitored, and the definition of assumptions and performance metrics for each of the assignments. This process formed the basis for the creation of a computational tool, called MDTMoodle, which provided the visualization of distance tutors' performances, using the concepts of traffic lights.
\end{abstract}

Keywords: Distance learning; Distance tutoring; Tutor monitoring. 


\section{Introdução}

A EaD vem ganhando importância no cenário nacional da educação nos últimos anos, tendo sido alvo de investimentos tanto por parte do setor privado como do setor público no Brasil. Pode-se constatar pelo Relatório Analítico da Aprendizagem a Distância o crescimento do número de matrículas no Brasil. No ano de 2009, havia cerca de 528.320 matrículas em cursos de EaD, já, em 2011, esse número cresceu para 3.589.373 matrículas (ABED, 2013).

Outro fato em que se confirma a importância da EaD, no contexto brasileiro, foi a implantação do sistema UAB, através do Decreto no 5.800, de 8 de junho de 2006, como também pela quantidade de cursos ofertados e matrículas que crescem a cada ano (BRASIL, 2006). Organizacionalmente, o funcionamento da UAB ocorre através de uma parceria entre os entes federativos, união, estados e munícipios, em que cada um fica responsável por uma parte do processo. A oferta dos cursos a distância ocorre em Polos de Apoio Presencial, sendo os munícipios responsáveis por proporcionar toda sua infraestrutura física para que sejam credenciados a ofertarem cursos a distância (BRASIL, 2006).

As participações da União e dos Estados ocorrem através das universidades públicas e dos Institutos Federais de Educação, Ciência e Tecnologia (IF) que ofertam os cursos, no papel de uma instituição de ensino superior (IES).

Os cursos a distância têm como principais atores: alunos, tutores, professores e coordenadores. As interações entre esses integrantes são realizadas através de sistemas computacionais denominados Ambientes Virtuais de Aprendizagem (AVAs). É o tutor quem se relaciona com os alunos, realizando mediação pedagógica (Bentes, 2009). Sobre as atribuições do tutor a distância, Ronchi et al. (2012) expandem-nas para englobar novas atribuições: estimular a aprendizagem ativa, estimular a autoaprendizagem, facilitar a aprendizagem; facilitar o aprendizado colaborativo; demonstrar comportamento interpessoal; esclarecer dúvidas nos fóruns dentre outras.

O documento de Referenciais de Qualidade para Educação Superior a Distância, publicado pelo Ministério da Educação (MEC), estabelece as ações que um tutor deve desempenhar em cursos regulamentados, nos processos de ensino-aprendizagem, juntamente com os discentes (BRASIL, 2007a). Dessa forma, percebe-se a importância 
do tutor a distância e o quanto ele afeta diretamente a qualidade desse tipo de ensino. Isso é reforçado pelo $\mathrm{MEC}$, que recomenda às instituições de EaD uma avaliação permanente e constante que envolva todos os seus atores (BRASIL, 2007b).

Todavia, a atividade de realizar o acompanhamento de suas atribuições não ocorre de forma fácil, uma vez que as atividades do tutor produzem um grande número de interações, dados e variáveis a serem consideradas. Ronchi et al. (2012) discutem sobre a diversidade de variáveis implicadas em acompanhamento dos tutores nos cursos a distância, enumerando 25 delas e agrupando-as em três dimensões: características pessoais, habilidades técnicas e atribuições a desempenhar.

Portanto, seria de grande importância para gerência, coordenação ou supervisão na EaD contar com o apoio de métodos e ferramentas que possibilitem a visualização de métricas de desempenho das atribuições dos tutores a distância, possibilitando um melhor acompanhamento das atividades de tutoria.

São vários os trabalhos relevantes relacionados à automatização do acompanhamento de tutorias. Rocael Pastor et al. (2010) atestaram que, na Universidade Nacional de Educação a Distância da Espanha (UNED), foi possível, dentro do AVA, obter acesso a dados estatísticos que possibilitaram detectar uma relação entre as notas dos alunos e o desempenho de um determinado tutor. Moniz (2010) idealizou uma ferramenta de gestão do Moodle, a GMoodle. O propósito desta ferramenta era armazenar dados do Moodle com o intuito de emitir relatórios gerenciais e proporcionar maior acompanhamento dos cursos. Almeida et al. (2011) analisaram os dados coletados sobre a atuação de um tutor em uma turma de EaD, formada por 50 alunos, em uma universidade pública que utilizou o TIDIA-AE (Tecnologia da Informação no Desenvolvimento da Internet Avançada - Aprendizado Eletrônico - http://www.tidia-ae.usp.br) como AVA. Esses autores relatam a carência de mecanismos automatizados que contribuam com a supervisão dos tutores nos cursos a distância. Felipe (2013) propõe um instrumento computacional para acompanhamento da interação no AVA dos cursos de graduação a distância da UABUniversidade do Estado do Ceará, denominado MOODLE Monitor.

Nesse contexto, o objetivo geral desta pesquisa é definir um método e construir uma ferramenta computacional que permitam, através de métricas quantitativas, acompanhar o desempenho das atribuições dos tutores a distância num ambiente 
virtual de aprendizagem de forma fácil através de semáforos. Como escopo, optou-se em aplicar a metodologia e a ferramenta num curso a distância ofertado pelo programa UAB em uma IES (Instituição de Ensino Superior).

Os objetivos específicos são: a) Identificar premissas sobre as atribuições dos tutores a distância; b) Obter informações sobre tutoria na base de dados do programa UAB na IES pesquisada; c) Discutir e executar análises quantitativas das atribuições de tutoria sob a ótica da equipe de supervisão e d) Prover aos supervisores de tutoria uma forma de visualização dos desempenhos dos tutores a distância.

\section{Metodologia}

Esta pesquisa teve dois momentos.

Inicialmente, no primeiro momento da pesquisa, utilizou-se de uma abordagem qualitativa, cujo foco foi compreender as atribuições dos tutores a distância no contexto da UAB na IES escolhida, aqui denominada IES $X$.

Quanto ao enquadramento do tipo de pesquisa, nesse primeiro momento, o estudo contém características de uma pesquisa-ação, uma forma de pesquisa qualitativa que busca modificar o ambiente que está sendo estudado através da ação do pesquisador (Wainer, 2007). Ao final desse momento, foi desenvolvido uma ferramenta computacional com intuito de facilitar o trabalho de supervisão de tutoria a distância no curso ofertado pelo programa UAB da IES X.

No segundo momento, a pesquisa utilizou uma abordagem quantitativa. Nessa fase do trabalho, foram realizadas análises e comparações dos resultados obtidos, através de gráficos do desempenho dos tutores a distância em cada uma das atribuições.

A coleta de dados nesse processo metodológico foi apoiada em: (1) análise documental em que foram investigados vários documentos como, por exemplo, projetos pedagógicos de curso, portarias, resoluções, apresentações e dados de levantamentos internos; (2) pré-testes dos questionários; (3) Aplicação dos questionários; (4) reuniões e entrevistas individuais e em grupo com a equipe de supervisão de tutoria a distância do curso dado pela UAB na IES X. 


\section{Resultados e discussão}

Após haver descrito à equipe de supervisão de tutoria da IES $X$ todo o processo metodológico a ser seguido neste estudo, iniciou-se o processo de definição de premissas e métricas de desempenho dos tutores a distância que deu suporte à criação da ferramenta computacional denominada Monitor de Desempenho do Tutor a Distância no MOODLE (MDTMoodle), que teria por meta final monitorar, através de semáforos, o desempenho dos tutores em suas atribuições.

O processo de coleta de dados para definição de métricas de desempenho dos tutores a distância foi dividido em três etapas: (i) definição das atribuições desempenhadas; (ii) classificação das atribuições; (iii) definição das premissas e métricas de desempenho.

Na primeira etapa, foi utilizado o Edital de Seleção de Tutores a Distância para o programa UAB da IES pesquisada. Esse edital serviu como instrumento de definição das atribuições dos tutores a distância. O edital lista objetivamente 18 atribuições que esse tipo de tutor deve exercer e encontra-se de acordo com as orientações dos órgãos de fomento, como a Coordenação de Aperfeiçoamento de Pessoal de Nível Superior (CAPES) e o MEC. Considerou-se que o referido edital é uma espécie de contrato formal de trabalho entre a instituição e a pessoa que irá desempenhar a tutoria.

Após a definição das atribuições de um tutor a distância, deu-se início a etapa de classificação de cada uma dessas atribuições. Para isso, foi definida uma estrutura de fácil compreensão para a equipe de gestão do curso pesquisado dividida em dois critérios: (1) "Meio de Execução" e (2) "Aspecto de Acompanhamento". O primeiro está relacionado ao uso do Moodle ou não para a execução da atribuição e o segundo, vinculado à forma de acompanhamento da mesma. Após a aplicação dos questionários, foram considerados para o estudo nove atribuições (ver Quadro 1). 
Quadro 1: Atribuições de tutoria a distância com interações no MOODLE e com aspecto de acompanhamento quantitativo.

\begin{tabular}{|c|c|}
\hline No & ATRIBUIÇÃO \\
\hline 10 & diariamente a disciplina. \\
\hline 11 & $\begin{array}{l}\text { Responder aos alunos no máximo em } 24 \text { horas. Realizar correção dos } \\
\text { trabalhos acadêmicos, em no máximo sete dias, além dos trabalhos de } \\
\text { recuperação paralela e exames presenciais dos alunos. }\end{array}$ \\
\hline 12 & $\begin{array}{l}\text { Manter-se em permanente comunicação com o professor formador, tutor } \\
\text { presencial e, acima de tudo, com os estudantes durante toda a disciplina. }\end{array}$ \\
\hline 13 & $\begin{array}{l}\text { Ter disponibilidade de } 20 \text { horas semanais, das quais } 12 \text { horas serão } \\
\text { destinadas ao atendimento de alunos em sistema on-line. As oito horas } \\
\text { semanais restantes serão destinadas a estudos e reuniões (reuniões } \\
\text { pedagógicas com o professor formador e/ou com o coordenador de } \\
\text { tutoria). }\end{array}$ \\
\hline 14 & orrigir atividades e provas. \\
\hline 15 & idades virtuais e presenciais. \\
\hline 16 & $\begin{array}{l}\text { Identificar alunos com dificuldade de acesso ou com baixo índice de } \\
\text { participação na disciplina e tomar as devidas providências para o seu } \\
\text { retorno ao curso. }\end{array}$ \\
\hline 17 & $\begin{array}{l}\text { Apresentar relatórios das atividades realizadas, presenciais e virtuais de } \\
\text { acordo com a solicitação da coordenação de tutoria. }\end{array}$ \\
\hline 18 & $\begin{array}{l}\text { Estabelecer contato permanente com os alunos e mediar as atividades } \\
\text { discentes. }\end{array}$ \\
\hline
\end{tabular}

Ao final do processo de classificação de tutoria a distância, foram desconsiderados quatro das nove atribuições. São elas: 14, 15, 16 e 17 do Quadro 1. Daí passou-se para a última etapa que consistiu na definição de premissas e métricas de cada uma das cinco atribuições restantes. As métricas definitivas encontram-se no Quadro 2. 
Quadro 2: Métricas definitivas.

\begin{tabular}{|c|l|}
\hline No ATRIBUIÇÃo & \multicolumn{1}{c|}{ MÉTRICA } \\
\hline 10 & $\begin{array}{l}\text { Percentual dos dias em que o tutor a distância acessou e } \\
\text { interagiu na disciplina em relação ao total de dias da } \\
\text { disciplina. }\end{array}$ \\
\hline 11 & $\begin{array}{l}\text { Percentual de solicitações atendidas dentro do prazo pelo } \\
\text { tutor a distância em relação ao número total de solicitações. }\end{array}$ \\
\hline 12 & $\begin{array}{l}\text { Média de frequência de contatos com toda a turma ao longo } \\
\text { da disciplina. }\end{array}$ \\
\hline 13 & $\begin{array}{l}\text { Percentual de tempo que o tutor passou on-line em relação } \\
\text { ao número total de horas on-line de atendimento que ele } \\
\text { deveria ter tido na disciplina. }\end{array}$ \\
\hline 18 & $\begin{array}{l}\text { Percentual do número de dias em que houve contato com a } \\
\text { turma em relação ao total de dias em que a disciplina } \\
\text { ocorreu. }\end{array}$ \\
\hline
\end{tabular}

Após a definição das premissas e métricas estabelecidas quanto aos dados coletados, foi iniciada a construção do MDTMoodle. Levando em conta os conceitos de Engenharia de Software, a criação do MDTMoodle foi dividida em quatro etapas: Análise, Projeto, Implementação e Teste. A Figura 1 mostra a tela do MDTMoodle.

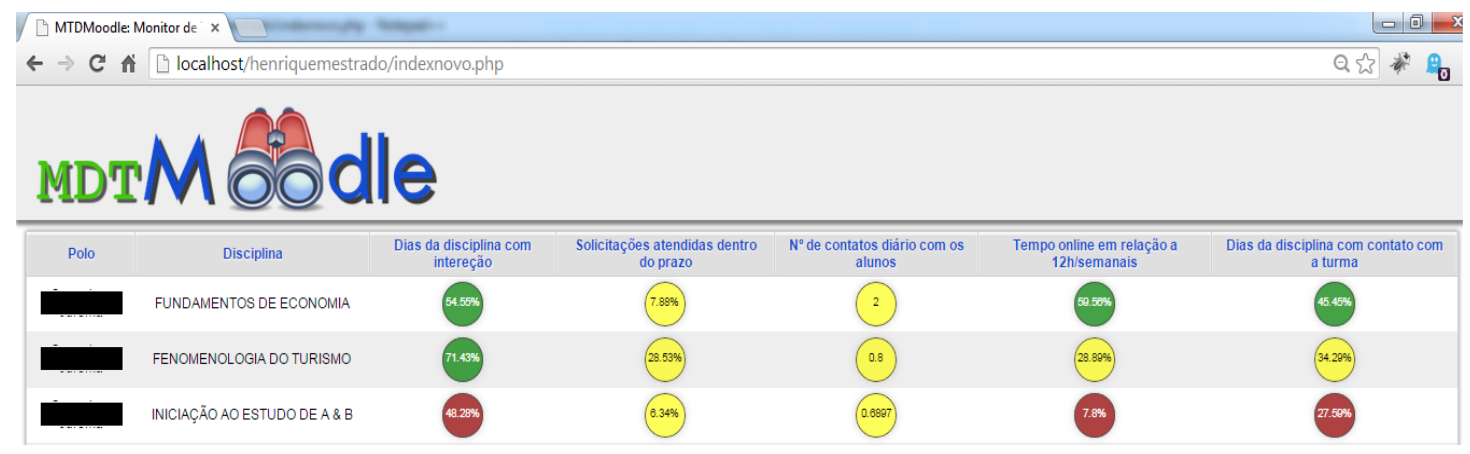

Figura 1: Visualização do desempenho dos tutores a distância no MDTMoodle.

Após a coleta de dados realizada pelo MDTMoodle, chegou-se aos desempenhos de cada atribuição nas 81 tutorias do primeiro semestre do curso analisado.

Os resultados da atribuição No 10 mostram que 71,60\% dos tutores acessaram e realizaram alguma ação em mais de $70 \%$ dos dias da disciplina, porém somente um 
tutor conseguiu acessar em todos os dias. Já os resultados obtidos na atribuição No 11 mostram que a maioria dos tutores teve um fraco desempenho. Os dados mostram que $96,29 \%$ dos tutores não conseguiram atender 50\% das solicitações dos alunos dentro do prazo estabelecido. Para a atribuição No 12, relativa à comunicação entre tutor e alunos, percebe-se, analisando o gráfico de dispersão na Figura 18, que 41,97\% dos tutores a distância tiveram em média menos de um contato diário com os alunos durante o período da disciplina. Essa atribuição mostra que, em uma grande parte da disciplina, houve uma baixa comunicação com a turma. Nos resultados relacionados ao desempenho dos tutores a distância na atribuição No 13, 46,91\% dos tutores conseguiram ficar on-line no sistema menos de seis horas semanais, ou seja, menos de $50 \%$ do tempo estabelecido de 12 horas semanais. Apesar desse baixo índice, observase em algumas disciplinas que os tutores ficaram 12 horas ou mais por semana no atendimento on-line aos alunos. Analisando os resultados obtidos na atribuição de No 18 , menos de um terço $(30,86 \%)$ dos tutores conseguiram manter contato com a turma em mais de $50 \%$ dos dias da disciplina. Nota-se, nesses resultados, que muitos tutores $(69,14 \%)$ passam mais da metade dos dias da disciplina sem se comunicar com a turma.

Realizou-se um cálculo de correlação linear de Pearson entre os resultados obtidos nas cinco atribuições monitoradas, totalizando 10 cálculos de correlação. Os cálculos mostraram que três correlações apresentaram um nível de intensidade entre as variáveis (no caso são as atribuições) moderado.

Analisando os resultados obtidos do cálculo entre as atribuições 11 e 18, observou-se um nível de correlação moderada $(0,417)$, atestando que quanto mais o tutor responde às solicitações dos alunos dentro do tempo estabelecido mais estabelece contato com os mesmos. Outra correlação moderada $(0,322)$ ocorreu entre as atribuições 12 e 13, comprovando que se o tutor passa mais tempo em contato com os alunos, mais tempo fica on-line e vice-versa. A terceira correlação moderada $(0,351)$ foi entre a atribuição 13 e 18 , atestando que, se o tutor passa um maior tempo on-line, ele se comunica mais com os alunos. 


\section{Conclusão}

O foco desta pesquisa foi a tutoria realizada a distância, através do MOODLE, nos cursos da UAB. Foram efetuadas uma coleta de dados e uma análise de dados de tutoria de um curso à distância do programa UAB.

Primeiramente, foi concebida uma ferramenta computacional (MDTMoodle), baseada em um processo de coleta de dados para definição de premissas e métricas para o monitoramento das atividades do tutor a distância. Ele, ainda, pode funcionar como elemento facilitador para a equipe que executa a gestão dos tutores, já que permite, através da visualização de semáforos, um acompanhamento quantitativo do desempenho dos tutores a distância.

Outra contribuição do processo é a identificação das atribuições que apresentam maiores dificuldades de execução pelos tutores a distância, possibilitando, assim, o planejamento de medidas que amenizem essas dificuldades.

Por fim, ainda foi observado que a metodologia fornece insumos para uma ferramenta gerencial de controle e monitoração a ser desenvolvida e utilizada em maior envergadura, podendo ser operacionalizada em diferentes cursos de EaD.

Esse trabalho de pesquisa possibilita outros trabalhos futuros como: definir uma classificação para o desempenho mínimo de cada função do tutor, ampliar o código fonte, adaptação do MDTMoodle com um plug-in para o Moodle e realizar novas coletas de dados em outras instituições e comparar seus resultados.

\section{Agradecimentos}

Ao IFCE, por viabilizar a realização desta pesquisa e disponibilização de dados. À Faculdade Católica Rainha do Sertão, pelo incentivo e suporte financeiro à publicação deste artigo.

\section{Referências bibliográficas}

ABED. (2013). Relatório Analítico da Aprendizagem a Distância no Brasil 2012. ABED Associação Brasileira de Educação a Distância Curitiba: Ibpex. 
Almeida, A.; Pimentel E. P.; Stiubiener I. (2011). Estratégias para o Monitoramento de Ações de Tutoria na Educação a Distância. In: Congresso Brasileiro de Ensino Superior a Distância. Ouro Preto.

Bentes, R. de F. (2009). A Avaliação do Tutor. In: Litto, F. M.; Formiga, M. Educação a Distância: o estado da arte. São Paulo: Pearson. p. 166-170.

Brasil. (2006). MEC - Decreto no 5.800, de 08 de junho de 2006. Dispõe sobre o Sistema Universidade Aberta do Brasil - UAB. Disponível em: http://uab.capes.gov.br/images/stories/downloads/legislacao/decreto5800.pdf

Brasil. (2007a). Secretaria de Educação a Distância - Ministério da Educação. Referenciais de Qualidade para Educação Superior a Distância. Brasília. Disponível em: http://portal.mec.gov.br/seed/arquivos/pdf/legislacao/ refEaD1.pdf

Brasil. (2007b). MEC. Portaria Normativa no 2, de 10 de janeiro de 2007. Disponível em:

http://uab.capes.gov.br/images/stories/downloads/legislacao/portaria normativ a 22007.pdf

Felipe, M. W. F. (2013). MOODLE Monitor: um instrumento computacional para acompanhamento da interação no ambiente virtual de aprendizagem dos cursos de graduação a distância da UAB/UECE. Dissertação (Mestrado) - Mestrado Profissional em Computação Aplicada - MPCOMP. Universidade Estadual do Ceará. Fortaleza.

Moniz, Lino Vaz. (2010). GMOODLE: ferramenta de gestão do MOODLE. Disponível em: http://www.badiu.net

Rocael Pastor, R.; Hernández, R.; ROS, Salvador; Read, T.; Castro, M.; (2010). A Complex Tutoring System for E-learning: new evaluation model. IEEE FIE Conference.

Ronchi, S. Haidar; Ensslin, S. R.; Ensslin L.; Petri S. M. (2012). Avaliação de desempenho da tutoria de educação a distância: um estudo da produção científica internacional para identificação das variáveis a comporem um modelo 
de avaliação, Revista Perspectivas Contemporâneas, Campo Mourão, v. 7, n. 2, p. 27-52. ISSN: 1980-0193.

Wainer, J. (2007). Métodos de Pesquisa Quantitativa e Qualitativa para a Ciência Computação. In T. Kowaltowski e K. Breitman (Org.) SBC, pp. 221-262. 